\title{
The cleistogamy of the superwoman1-cleistogamy1 mutation is sensitive to low temperatures during the lodicule-forming stage
}

\author{
Shinnosuke Ohmori ${ }^{1,5)}$, Setsuo Koike ${ }^{2)}$, Takami Hayashi ${ }^{2,3)}$, Tomoya Yamaguchi ${ }^{2,6)}$, Makoto Kuroki ${ }^{3,4)}$ \\ and Hitoshi Yoshida*4,7) \\ 1) Hokuriku Research Center, NARO (National Agriculture and Food Research Organization) Agricultural Research Center, 1-2-1 Inada, \\ Joetsu, Niigata 943-0193, Japan \\ 2) Tohoku Agricultural Research Center, NARO, 4 Akahira, Shimo-kuriyagawa, Morioka, Iwate 020-0198, Japan \\ 3) Hokkaido Agricultural Research Center, NARO, 1 Hitsujigaoka, Toyohira, Sapporo, Hokkaido 062-8555, Japan \\ 4) Institute of Crop Science, NARO, 2-1-18 Kannondai, Tsukuba, Ibaraki 305-8518, Japan \\ 5) Present address: Headquarter, NARO, 3-1-1 Kannondai, Tsukuba, Ibaraki 305-8517, Japan \\ 6) Present address: Agriculture, Forestry and Fisheries Research Council, 1-2-1 Kasumigaseki, Chiyoda, Tokyo 100-8950, Japan \\ 7) Present address: Institute of Agrobiological Sciences, NARO, 2-1-2 Kannondai, Tsukuba, Ibaraki 305-8602, Japan
}

We reported previously that the rice (Oryza sativa L.) cleistogamous mutation superwoman1-cleistogamy1 (spw1-cls 1 ) was applicable to inhibit outcrossing between genetically modified varieties and their relatives, which causes pollen-mediated gene flow or disturbance of line purity. The cleistogamy of spwl-cls 1 is caused by decreased protein-protein interactions between the mutant SPW1 and its partner proteins. Importantly, these interactions are restored under low-temperature conditions, but whether the cleistogamy of spwl-cls 1 is affected by this phenomenon was unclear. In this study, we cultivated spwl-clsl in various regions of Japan and confirmed that its flowers opened at low temperatures. Moreover, we compared the morphology of a series of lodicules generated at various temperatures. The results indicated that the cleistogamy of $s p w 1-c l s 1$ is thermosensitive and is gradually disturbed as the temperature decreases. This was correlated with the protein interaction pattern of the mutant SPW1 as reported previously. Then, we revealed the critical period for the lowtemperature-induced instability of the phenotype of spwl-cls1 and examined the effect of daily temperature changes on cleistogamy. The results may facilitate simulation of the phenotype of spw1-cls1 at various temperatures and the prediction of regions where the cleistogamy of $s p w 1-c l s 1$ can be stably used to inhibit outcrossing.

Key Words: genetically modified rice, outcrossing, gene flow, cleistogamy, flower opening, lodicule, thermosensitive.

\section{Introduction}

Various genetically modified (GM) crops are cultivated worldwide, and the area of GM crop cultivation is expected to expand in the future (ISAAA 2016). Since the commercial cultivation of GM crops was initiated, outcrossing between GM crops and their relatives (weeds, wild species, and non-GM conventional crops) is a concern because foreign genes introduced into GM crops may spread elsewhere (Daniell 2002, Gressel 2012, Hüsken et al. 2010, MallorySmith and Zapiola 2008). This pollen-mediated gene flow

Communicated by Motoyuki Ashikari

Received March 7, 2018. Accepted May 31, 2018.

First Published Online in J-STAGE on August 28, 2018.

*Corresponding author (e-mail: yocida@affrc.go.jp) may reduce environmental biodiversity. To inhibit this, gene-containment measures (e.g., male sterility, setting temporal/spatial barriers between pollen donors and recipients, etc.) have been examined in various crops.

Outcrossing between GM crops and relatives may reduce the quality of GM crops. GM crops will lose favorable traits if pollinated with the pollen of relatives. Maintaining the genetic purity of special traits in GM crops is important, particularly when they are used for medical applications or industrial raw materials. Sustainable cultivation of GM crops requires control of outcrossing.

Although GM rice (Oryza sativa L.) is cultivated in a small land area, it is important to establish measures to inhibit outcrossing between GM rice and its relatives in preparation for increased cultivation. Under experimental conditions, the outcrossing rate of various cultivated rice varieties 
(including GM rice) is low (Endo et al. 2009, Messeguer et al. 2001, Rong et al. 2007, 2012). This is because pollination of almost all rice flowers occurs just before flower opening, and fertilization is complete when the pollen grains from foreign flowers reach the stigma (Hoshikawa 1989). However, the outcrossing rate is increased by low temperatures, which cause male sterility and decrease the rate of self-fertilization (Sato and Yokoya 2008, Tanno et al. 2011), and by the shape and size of floral organs (Kato and Namai 1987a, 1987b, Marathi and Jena 2015). Certain wild rice varieties have a high outcrossing rate because of their high cross-affinity for cultivated rice (Phan et al. 2012).

Cleistogamy is defined as fertilization without flower opening. In a cleistogamous flower, pollen does not leave the flower and stigmas are not exposed to pollen grains from other flowers; in other words, outcrossing is inhibited. Moreover, cleistogamy is compatible with cultivated rice because it is an autogamous crop. In fact, cleistogamy is usually observed in rice on rainy flowering days (Hoshikawa 1989). Introduction of cleistogamy to rice would protect GM rice from transgene flow by pollen dispersal and maintain the purity of rice cultivars and lines.

Among the rice cleistogamous mutants identified to date, superwoman1-cleistogamyl (spw1-cls1) is the most well-characterized and practical (Maeng et al. 2006, Nagao and Takahashi 1954, Ni et al. 2014, Yoshida et al. 2007). This mutant was isolated from a mutagenized population derived from 'Taichung 65' (T65), and a single-base substitution was identified in the SUPERWOMAN1 gene. This gene encodes a rice class B MADS-box transcription factor and specifies the identities of lodicules (petal-equivalent organs in grass species) and stamens (Nagasawa et al. 2003). The single-base substitution causes an amino-acid substitution (isoleucine at the 45th amino acid position to threonine; I45T) in the $\beta$-strand of the MADS-box domain, which is related to the interactions of MADS-box proteins (Han et al. 2003, Norman et al. 1988). Yoshida et al. (2007) reported that the ability of mutant SPW $1^{\mathrm{I} 55 \mathrm{~T}}$ protein to interact with MADS2 and MADS4 is reduced. Although this defect in protein-protein interactions does not affect stamen development, the lodicules lose normal identity and are homeotically transformed into organs resembling the marginal region of the palea (mrp). Because this organ does not have swelling function, $s p w 1-c l s 1$ shows a cleistogamous phenotype. We reported previously that cleistogamy of $s p w 1-c l s 1$ inhibits outcrossing in paddy fields without significant loss of its commercial value. Moreover, this cleistogamous phenotype can be introduced to other cultivars or lines by artificial crossing and selection using a DNA marker designed to recognize spw1-cls1 (Ohmori et al. 2012). Using a yeast twohybrid system, Yoshida et al. (2007) demonstrated that the ability of SPW $1^{145 T}$ to interact with MADS2 is significantly restored as the temperature decreases. Because $s p w 1-c l s 1$ has not been cultivated at low temperatures, whether this restoration affects its cleistogamous phenotype is unclear.

In this study, we performed experimental cultivation of spwl-cls 1 under various climatic conditions in Japan and examined its flower opening and lodicule phenotype. Next, to explore the temperature sensitivity of $s p w 1-c l s 1$ cleistogamy, we cultivated spwl-cls 1 under various controlledtemperature conditions using growth chambers and artificial climate rooms. By investigating the lodicule morphology under these conditions, we found the structural alteration of lodicules that causes flower opening of spwl-cls1. By scheduled low-temperature treatments, we also revealed that the lodicule-forming stage is the most temperaturesensitive period of panicle development in spw1-clsl, and estimated the effect of daily temperature change on its cleistogamy. Our study links previous molecular analyses with the cleistogamy phenotype of $s p w 1-c l s 1$, and provides important insight into the potential of $s p w 1-c l s 1$ to inhibit outcrossing.

\section{Materials and Methods}

\section{Plant materials}

The rice cleistogamous mutant $s p w 1-c l s 1$ was isolated from an $\mathrm{M}_{2}$ population mutagenized with $N$-methyl- $N$ nitrosourea derived from a japonica rice cultivar, T65 (Ohmori et al. 2012, Yoshida et al. 2007).

\section{Field cultivation}

Field cultivation of spw1-cls 1 was conducted at the following eight locations in Japan: Sapporo (Hokkaido Agricultural Research Center, NARO), Morioka (Tohoku Agricultural Research Center, NARO), Daisen (Tohoku Agricultural Research Center, Daisen Research Station, NARO), Osaki (Miyagi Prefectural Furukawa Agricultural Experiment Station), Tsukuba (Institute of Crop Science, NARO), Joetsu (Central Region Agricultural Research Center, Hokuriku Research Center, NARO), Fukuyama (Western Region Agricultural Research Center, NARO), and Itoman (Kyushu Okinawa Agricultural Research Center, Itoman Resident Office) in 2009 and 2010 (Fig. 1, Table 1). All cultivations were performed under standard conditions.

Two or three days after the heading date $(50 \%$ heading, HD), we categorized the flower-opening frequency (FOF) of spwl-cls 1 at each cultivation site into four classes (Table 1). Because different co-experimenters had to judge the frequency of flower opening at each cultivation site, we employed this FOF as a simple method to reduce errors in judges. Daily mean temperature was measured at each cultivation site. Only in Daisen, we employed the data of the nearest AMeDAS point (Omagari) from the Japan Meteorological Agency website (http://www.jma.go.jp/). The 30-day mean temperature before HD (30MT) was calculated from the daily data.

To compare lodicule phenotypes, we collected spikelet samples from booting-stage panicles, and directly observed fresh flowers by scanning electron microscopy (VE-8000; Keyence Corporation, Osaka, Japan). T65 was used as the wild type (WT) for lodicule comparison. 


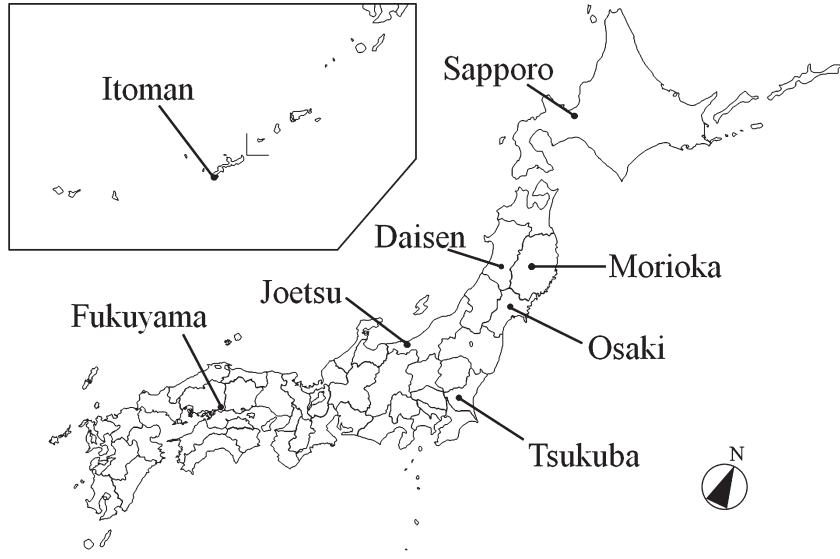

Fig. 1. Map of the experimental cultivation sites.

Table 1. Flower-opening frequency, heading date, and 30-day mean temperature before the heading date of $s p w 1-c l s 1$

\begin{tabular}{lcccccccc}
\hline \hline \multirow{2}{*}{$\begin{array}{c}\text { Cultivation } \\
\text { site }\end{array}$} & \multicolumn{4}{c}{2009} & & \multicolumn{3}{c}{2010} \\
\cline { 2 - 4 } \cline { 6 - 8 } \cline { 6 - 8 } & FOF* & HD & 30MT $\left({ }^{\circ} \mathrm{C}\right)$ & & FOF & HD & 30MT $\left({ }^{\circ} \mathrm{C}\right)$ \\
\hline Sapporo & $\mathrm{A}$ & $9 / 7$ & 19.5 & & $\mathrm{~B}$ & $8 / 25$ & 22.9 \\
Morioka & $\mathrm{B}$ & $8 / 10$ & 22.5 & & - & - & - \\
Daisen & $\mathrm{B}$ & $8 / 22$ & 24.1 & & - & - & - \\
Osaki & $\mathrm{B}$ & $8 / 19$ & 22.7 & & - & - & - \\
Joetsu & $\mathrm{B}$ & $8 / 16$ & 24.3 & & $\mathrm{~N}$ & $8 / 11$ & 27.4 \\
Tsukuba & $\mathrm{C}$ & $8 / 22$ & 25.6 & & $\mathrm{~N}$ & $8 / 21$ & 28.4 \\
Fukuyama & $\mathrm{N}$ & $8 / 19$ & 26.5 & & $\mathrm{~N}$ & $8 / 18$ & 29.4 \\
Itoman & $\mathrm{N}$ & $8 / 11$ & 29.5 & & - & - & - \\
\hline
\end{tabular}

FOF: flower-opening frequency. HD: heading date. 30MT: 30-day mean air temperature before heading date. ${ }^{*} \mathrm{~A}$ : almost all flowers open, B: less than half of flowers open, C: some flowers open, $\mathrm{N}$ : no flowers open.

\section{Analysis of spw1-cls1 phenotype according to temperature Preparation of plant materials}

To obtain spw1-cls1 individuals at the same panicle developmental stages, we employed the circular dense-culture method (Satake 1972). Twenty spwl-cls 1 seeds were planted in Wagner pots (1/5000a) and cultivated in a greenhouse under long-day conditions. All tillers were cut off whenever they emerged, leaving only the main stems. After the leaf age of the individuals reached $\sim 12.0$, a short-day treatment (12-h day) was started. Young panicle initiation (Fig. 2) was confirmed by dissecting one of the individuals in the pots and the pots were subjected to three temperature treatments (described later). To exclude genetic effects, we used a seed set produced by a single individual in each experiment. Plants showing bad growth or widely divergent heading dates (earlier or later) were not used.

\section{Temperature treatments}

Experiment 1: Effect of low temperatures on the lodicule phenotype of spw1-cls1

We prepared four growth chambers (MLR-350HT or MLR-350; Sanyo Electric Co., Ltd., Osaka, Japan) with temperatures of $30^{\circ} \mathrm{C} / 25^{\circ} \mathrm{C}, 30^{\circ} \mathrm{C} / 20^{\circ} \mathrm{C}, 25^{\circ} \mathrm{C} / 20^{\circ} \mathrm{C}$, and $20^{\circ} \mathrm{C} / 20^{\circ} \mathrm{C}$ (Table 2). Two pots of spwl-cls 1 were set in each growth chamber for 3 weeks. After temperature treat-

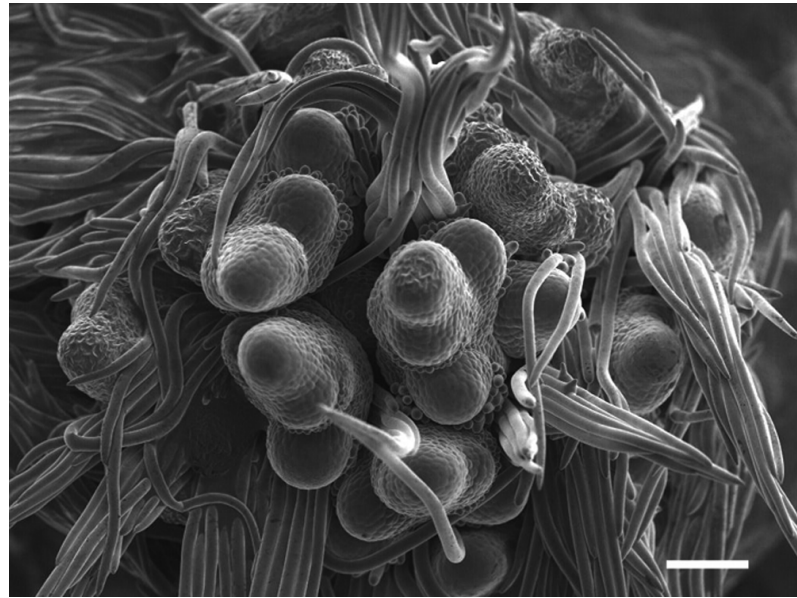

Fig. 2. A representative scanning electron micrograph of a confirmed young panicle before starting temperature treatment (secondary rachisbranch differentiation stage). Scale bar $=83.3 \mu \mathrm{m}$.

ments, all pots were returned to the greenhouse. Just before heading (booting stage), we sampled mature spikelets from each plant. After removing the lemmas, the lodicule length (from the base to the top) was measured under a stereomicroscope using digital calipers (CD-15PSX; Mitutoyo Corporation, Kanagawa, Japan). Cross sections were prepared from rice flowers as described previously (Kawakatsu et al. 2006, Ohmori et al. 2009), and tissues were stained with toluidine blue. As controls, we used spikelets of T65 and $s p w 1-c l s 1$ that were cultivated in a paddy field (Joetsu City, Niigata Prefecture) under natural conditions. These spw1-cls 1 showed a cleistogamous phenotype.

Experiment 2: Effect of panicle developmental stage on low-temperature-sensitive flower opening in $s p w 1$-cls 1

In preliminary experiments, the duration from young panicle initiation until the start of heading was about 4 weeks in spw1-cls1 (data not shown). Therefore, we divided this duration into four 7-day periods-I, II, III, and IV. We prepared two artificial climate rooms (custom order equipment, Construction No. K31-6389; Koito Electric Industries, Ltd., Shizuoka, Japan). One was set to $30^{\circ} \mathrm{C} / 25^{\circ} \mathrm{C}$ (light/dark period temperature, $12 \mathrm{~h}$ each) and used for the normal-temperature treatment, and the other was set to

Table 2. Lengths of lodicules according to temperature conditions

\begin{tabular}{|c|c|c|c|c|}
\hline Plot name & \multicolumn{2}{|c|}{$\begin{array}{l}\text { Diagram of daily temperature } \\
\text { treatment schedule }\end{array}$} & $\mathrm{n}=$ & $\begin{array}{l}\text { Mean length } \\
\text { of lodicules } \\
(\mathrm{mm})\end{array}$ \\
\hline \multirow[b]{2}{*}{$30^{\circ} \mathrm{C} / 25^{\circ} \mathrm{C}$} & Light period (12h) & Dark period (12h) & \multirow[b]{2}{*}{110} & \multirow[b]{2}{*}{$2.93 \pm 0.15 \mathrm{a}$} \\
\hline & $30^{\circ} \mathrm{C}$ & $25^{\circ} \mathrm{C}$ & & \\
\hline $30^{\circ} \mathrm{C} / 20^{\circ} \mathrm{C}$ & $30^{\circ} \mathrm{C}$ & $20^{\circ} \mathrm{C}$ & 125 & $2.80 \pm 0.21 \mathrm{~b}$ \\
\hline $25^{\circ} \mathrm{C} / 20^{\circ} \mathrm{C}$ & $25^{\circ} \mathrm{C}$ & $20^{\circ} \mathrm{C}$ & 110 & $2.04 \pm 0.17 \mathrm{c}$ \\
\hline $20^{\circ} \mathrm{C} / 20^{\circ} \mathrm{C}$ & 20 & & 105 & $1.57 \pm 0.29 \mathrm{~d}$ \\
\hline
\end{tabular}

$\mathrm{n}=$ number of measured lodicules. Mean \pm standard deviation. The same letters are not significantly different at $p<0.01$ by Scheffe's F-test. 
$24^{\circ} \mathrm{C} / 20^{\circ} \mathrm{C}$ and used for the low-temperature treatment. Next, we designed 12 low-temperature treatment schedules. Each schedule had a different low-temperature duration and timing (Table 3) and was named according to the timing of the low-temperature treatment. For example, in the LT-I schedule, the plants were treated with the low temperature in period I and transferred to the normal-temperature room for the other three periods. In the LT-I+III schedule, the low-temperature treatment was applied in periods I and III. In the LT-I+II+III+IV schedule, plants were subjected to low-temperature treatment in all four periods and plants in No-LT were not exposed to the temperature treatments. After each temperature treatment schedule, all pots were returned to the greenhouse.

Generally, when rice anthesis occurs, the stamens protrude outside of the flower by filament elongation through the gap between the lemma and palea. After finishing the anthesis, the lemma and palea close, and the withered anthers of the flower are left on the outside of the glumes (Hoshikawa 1989). However, this phenomenon does not occur when the flower is cleistogamous (Maeng et al. 2006, Ohmori et al. 2012, Yoshida et al. 2007). Therefore, we judged whether rice flowers had opened or not by determining whether anthers were adhering to the outside of the glumes. After heading initiation of each plant, we checked all flowers per panicle, and counted the number of flowers judged to be opened. The flower-opening rate (FOR) per panicle was calculated by dividing the number of opened flowers by the number of all flowers on the panicle. We ex- amined 6 to $19 \mathrm{spw1-cls1}$ panicles per temperature treatment to investigate the FOR. Because we conducted this experiment twice (exp. 2-1 and 2-2) with different seed sets at different times, each test result was subjected to statistical analysis separately.

Experiment 3: Effect of high-temperature pulse treatments on flower opening of spw1-cls 1

We conducted two tests (exp. 3-1 and 3-2) using various daily temperature cycle patterns. First, to evaluate the effect on the FOR of low temperatures in the dark period, two growth chambers (same as in Experiment 1) were set at $25^{\circ} \mathrm{C} / 25^{\circ} \mathrm{C}$ (light/dark period temperature, $12 \mathrm{~h}$ each; plot name, 25 -all) and $25^{\circ} \mathrm{C} / 18^{\circ} \mathrm{C}$ (plot name, 25-18) (Table 4). In exp. 3-2, to estimate the effect of temperature change in the daytime, four growth chambers were set as follows: $25^{\circ} \mathrm{C} / 18^{\circ} \mathrm{C}$ (same as $25-18$ ); for the other three treatments, 1,2 , and $4 \mathrm{~h} 30^{\circ} \mathrm{C}$ treatments were inserted into the middle of the light period (Table 4). The plots were named D30-0h, D30-1h, D30-2h, and D30-4h, according to the duration of the inserted $30^{\circ} \mathrm{C}$ treatments.

The spw1-cls 1 pots were placed in the growth chamber and exposed to each temperature cycle until completion of heading. We used the same method to collect FOR data as in Experiment 2 and examined 35 to 39 spwl-cls 1 panicles per plot to determine the FOR. The FOR data in exp. 3-2 were subjected to statistical analysis. The daily mean air temperature (DMT) was calculated from the setting temperature inside the growth chamber.

Table 3. Determination of the temperature-sensitive period of spwl-cls 1

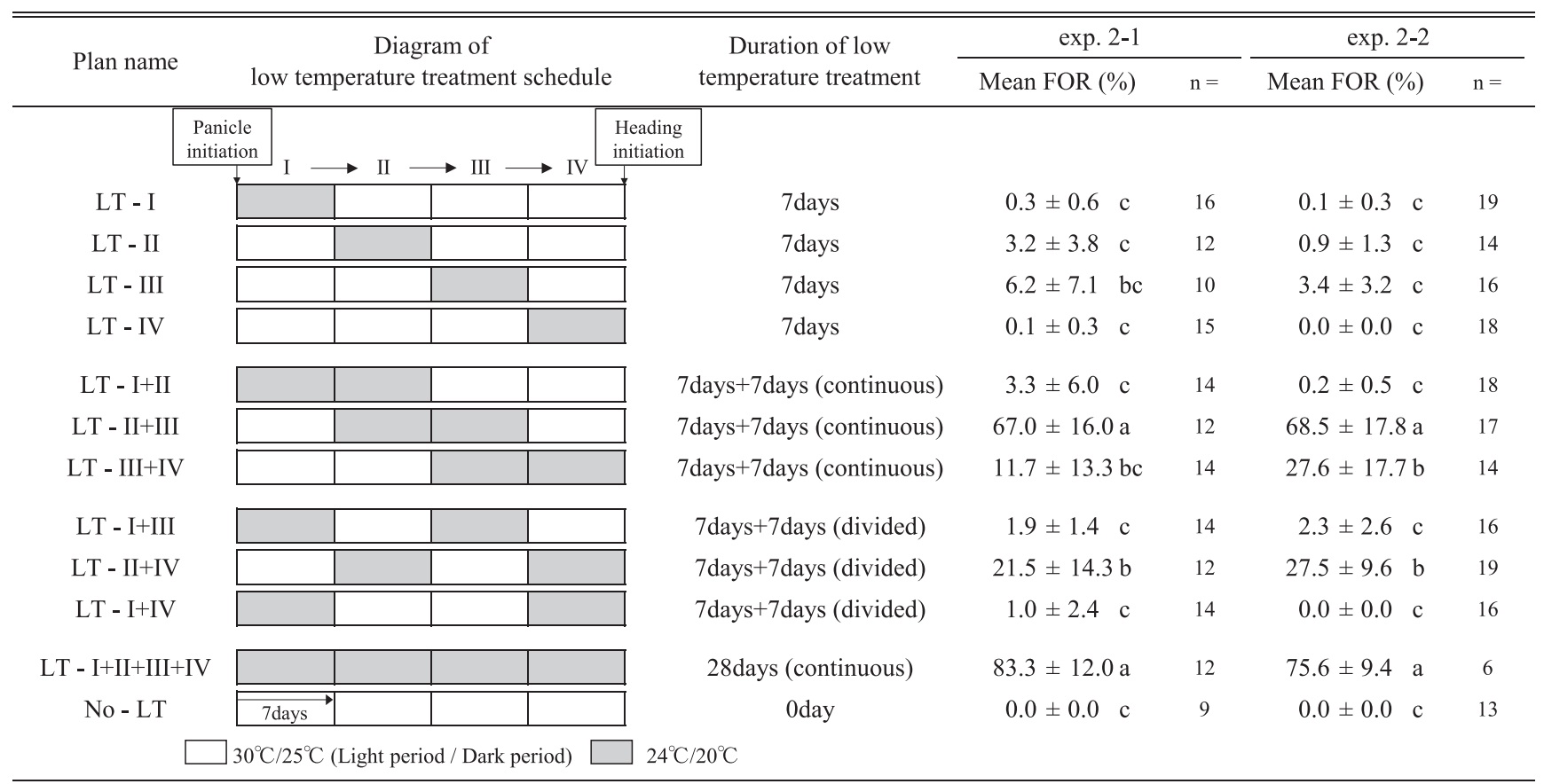

I, II, III, and IV are each 7 days long. Blue rectangles indicate low-temperature treatment, and white rectangles show no treatment. The light and dark periods were each $12 \mathrm{~h}$ long. FOR: flower-opening rate per panicle. $\mathrm{n}=$ number of panicles. Values are mean \pm standard deviation. Values with the same letter are not significantly different at $\mathrm{p}<0.01$ by Scheffe's F-test. 
Table 4. Effect of daytime temperature changes on flower opening of spwl-cls 1

\begin{tabular}{|c|c|c|c|c|c|c|c|}
\hline \multicolumn{2}{|c|}{ Plot name } & \multicolumn{3}{|c|}{$\begin{array}{l}\text { Diagram of } \\
\text { daily temperature treatment schedule }\end{array}$} & FOR $(\%)$ & \multirow[t]{2}{*}{$\mathrm{n}=$} & \multirow[t]{2}{*}{$\begin{array}{l}\text { DMT } \\
\left({ }^{\circ} \mathrm{C}\right)\end{array}$} \\
\hline \multirow{3}{*}{$\begin{array}{l}\vec{n} \\
\dot{m} \\
\dot{x}\end{array}$} & & Light perio & $(12 \mathrm{~h})$ & Dark period $(12 \mathrm{~h})$ & \multirow{3}{*}{$\begin{aligned} 5.0 & \pm 5.5 \\
55.3 & \pm 17.5\end{aligned}$} & & \\
\hline & 25 - all & & & & & \multirow{2}{*}{$\begin{array}{l}36 \\
35\end{array}$} & \multirow{2}{*}{$\begin{array}{l}25.0 \\
21.5\end{array}$} \\
\hline & $25-18$ & & & & & & \\
\hline \multirow{4}{*}{$\begin{array}{l}\text { r } \\
\text { ñ } \\
\dot{\vec{x}}\end{array}$} & D30-0h & & & & $49.9 \pm 14.6 \mathrm{a}$ & 37 & 21.5 \\
\hline & D30-1h & \begin{tabular}{l|l|}
$5.5 \mathrm{~h}$ & $1 \mathrm{~h}$ \\
\end{tabular} & $5.5 \mathrm{~h}$ & & $24.0 \pm 13.5 \mathrm{~b}$ & 39 & 21.7 \\
\hline & D30-2h & \begin{tabular}{l|l|}
$5 \mathrm{~h}$ & $2 \mathrm{~h}$ \\
\end{tabular} & $5 \mathrm{~h}$ & & $13.4 \pm 9.3 \mathrm{c}$ & 39 & 21.9 \\
\hline & D30-4h & $4 \mathrm{~h}$ & $4 \mathrm{~h}$ & & $0.3 \pm 0.7 \quad d$ & 38 & 22.3 \\
\hline & & $\square 25^{\circ} \mathrm{C}$ & 血程 & 50 & & & \\
\hline
\end{tabular}

FOR: flower-opening rate per panicle (mean \pm standard deviation). Values with the same letter are not significantly different at $\mathrm{p}<0.01$ by Scheffe's F-test. $\mathrm{n}=$ number of panicles. DMT: Daily mean air temperature of the growth chamber.

\section{Statistical analysis}

Statistical analysis was performed using Scheffe's F-test for multiple comparisons.

\section{Results}

Flower-opening frequency and lodicule morphology of spw1-cls1

The FOFs, HDs, and 30MTs are shown in Table 1. In 2009, Japan had a cooler-than-usual summer. In Sapporo, the FOF of $s p w 1-c l s 1$ was classified as A; that is, spw1-cls 1 flowers opened similarly to those of WT chasmogamous lines. The 30MT of this site was the lowest of all of the cultivation sites. In Morioka, Daisen, Osaki, and Joetsu, the FOFs were classified as B. The numbers of open flowers were significantly lower than in Sapporo. We observed a small number of open flowers in Tsukuba, and complete cleistogamy in Fukuyama and Itoman. We classified the FOF of Tsukuba as $\mathrm{C}$, and those of Fukuyama and Itoman as $\mathrm{N}$. The 30MT of Itoman was the highest of all of the cultivation sites.

We repeated the experimental cultivations in 2010, which had a very hot summer, at four cultivation sites. The FOF of Sapporo was classified as B. The number of open flowers was significantly decreased compared with those in the previous year. Because there were no open flowers in Joetsu, Tsukuba, and Fukuyama, the FOFs of these sites were classified as N. The 30MTs of 2010 were higher than those of 2009 at each cultivation site, and the FOFs of each site in 2010 were different from those in 2009, except for Fukuyama.

The lodicule morphology of each site in 2009 is shown in Fig. 3. The spw1-cls1 lodicules at Sapporo were comparatively short shape, similar to WT lodicules (Fig. 3A, 3B). In contrast, the lodicules of the sites with B FOFs were slender (Fig. 3C, only Joetsu is shown) compared with those of Sapporo, and the lodicules lengthened as the site locations shifted southwards (Fig. 3D, 3E). Moreover, the spw1-cls 1 lodicules at Itoman were elongated and the bottom part had lost its round shape (Fig. 3F). These transformed lodicules were similar to the palea-like organs of $s p w 1-1$ reported by Nagasawa et al. (2003). We found identical morphological changes in spw1-cls1 lodicules in 2010 (data not shown).

There was a clear correlation between the FOFs of spw 1 $c l s 1$ and the location of the cultivation sites. Although flower opening of $s p w 1-c l s 1$ was confirmed at northern cultivation sites, the FOFs were $\mathrm{N}$ at southern cultivation sites, where the 30MTs were higher. This trend was consistent with the morphological changes of spw1-cls1 lodicules. Annual changes in climate also affected the FOFs.

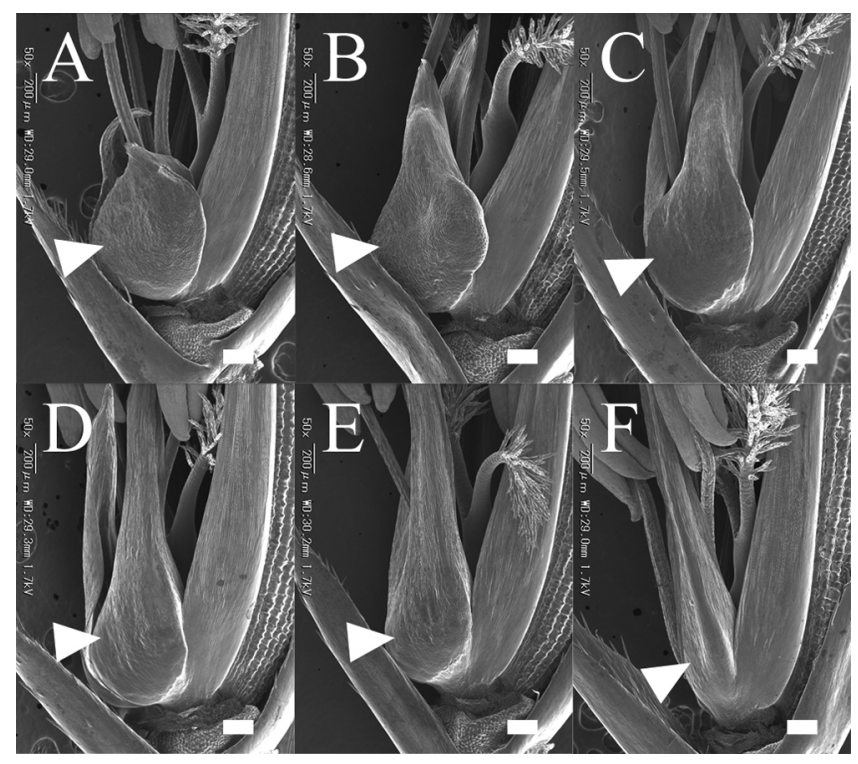

Fig. 3. Lodicule morphology of spwl-cls 1 cultivated in various regions of Japan in 2009. (A) Lodicule of WT(T65). (B) Lodicule of spw1-cls 1 cultivated in Sapporo. (C) Lodicule of spw1-cls 1 cultivated in Joetsu. (D) Lodicule of spwl-clsl cultivated in Tsukuba. (E) Lodicule of spwl-cls 1 cultivated in Fukuyama. (F) Lodicule of spw1-cls 1 cultivated in Itoman. White arrowheads indicate lodicules. Scale bar $=200 \mu \mathrm{m}$. 


\section{Response of spw1-cls1 phenotype to temperature}

Experiment 1: Effect of temperature on the appearance and internal structure of spw1-cls1 lodicules

Lodicules of $s p w 1-c l s 1$ are transformed to elongated organs resembling mrp, while normal rice lodicules are of a short scale shape (Fig. 4A, 4B; Ohmori et al. 2012, Yoshida et al. 2007). In this experiment, we compared the shape and internal structure of lodicules that formed under various temperature conditions.

In the highest-temperature treatment plot $\left(30^{\circ} \mathrm{C} / 25^{\circ} \mathrm{C}\right)$, lodicules of spw1-cls1 were strongly elongated and narrowed (Fig. 4C left end). The phenotype resembled that of $s p w 1-c l s 1$ cultivated in Itoman in 2009 (Fig. 3F). The mean lodicule length of this plot was $2.93 \mathrm{~mm}$ (Table 2), which was the longest of all of the plots. In the $30^{\circ} \mathrm{C} / 20^{\circ} \mathrm{C}$ plot, spw1-cls 1 lodicules were also severely elongated as similar to those of the $30^{\circ} \mathrm{C} / 25^{\circ} \mathrm{C}$ plot (Fig. $4 \mathrm{C}$ middle left). The mean lodicule length of this plot was $2.80 \mathrm{~mm}$. Although the difference in lodicule length between this and the highest-temperature plots was significant, it was small. spw1-cls 1 lodicules in the $25^{\circ} \mathrm{C} / 20^{\circ} \mathrm{C}$ plot condition were somewhat shorter than those of the two higher-temperature plots (Fig. 4C middle right). The mean lodicule length of this plot was $2.04 \mathrm{~mm}$. In the lowest-temperature plot $\left(20^{\circ} \mathrm{C} / 20^{\circ} \mathrm{C}\right)$, the lodicules were clearly short and similar to the lodicules at Sapporo in 2009 (Figs. 3B, 4C right end). The mean lodicule length of this plot was $1.57 \mathrm{~mm}$, which was the shortest of all of the plots. Damaged anthers were observed in this plot because of the low temperatures during stamen development.

Overall, lodicules of spwl-cls1 became shorter as the temperature decreased, and gradually came to resemble WT lodicules. This tendency was consistent with the results of experimental cultivation in various climatic regions in Japan.

The structures of lodicule and mrp in the WT differed markedly (Fig. 4D). The entire lodicule was stained blue by toluidine blue, and consisted of small parenchymal cells (PL, parenchyma of lodicule). Its vascular bundles were arranged in two rows. In contrast, the tissue of mrp was stained blue-green, and mainly comprised fibrous sclerenchymatous cells with a high density on the abaxial side (SM, sclerenchyma of mrp; Fig. 4D black arrow) and large, sponge-like parenchymal cells on the adaxial side (PM, parenchyma of mrp; Fig. 4D black arrowhead) (Hoshikawa 1989).

Almost all cross sections of $s p w 1-c l s 1$ lodicules formed

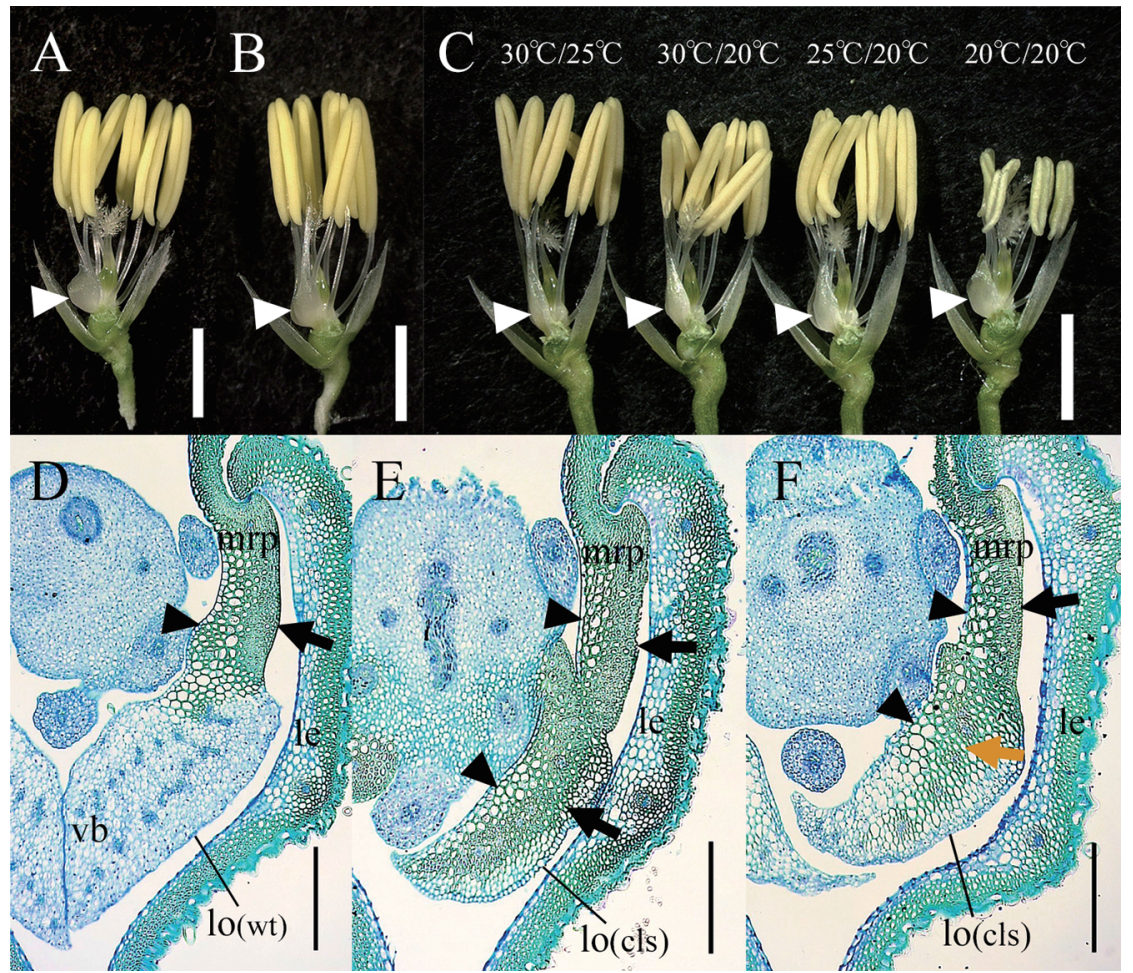

Fig. 4. Appearance and internal structure of spwl-cls1 lodicules formed under various temperature conditions. (A) Lodicule of WT(T65) cultivated in a paddy field at Joetsu. (B) Lodicule of spw1-cls 1 cultivated in a paddy field at Joetsu, showing a cleistogamous phenotype. (C) Lodicules of $s p w 1$-cls 1 cultivated under different temperature conditions in growth chambers. Numbers above spikelets are the air temperature conditions (light/dark periods). (A-C) Lemmas and paleae were removed from spikelets. White arrowheads indicate lodicules. Scale bar: $2.5 \mathrm{~mm}$. (D) Internal structure of WT lodicule and mrp. (E) Internal structure of spwl-cls 1 lodicule and mrp formed under $30^{\circ} \mathrm{C} / 25^{\circ} \mathrm{C}$ conditions. (F) Internal structure of spwl-cls 1 lodicule and mrp formed under $20^{\circ} \mathrm{C} / 20^{\circ} \mathrm{C}$ conditions. (D-F) Cross sections stained toluidine blue. Black arrows indicate sclerenchyma of mrp (SM). Black arrowheads indicate parenchyma of mrp (PM). Orange arrow indicates fibrous-parenchymatous intermediate tissue. Scale bar: $200 \mu \mathrm{m}$. lo(wt): lodicule of WT, lo(cls): lodicule of spw1-cls1, mrp: marginal region of palea, le: lemma, vb: vascular bundle. 
under $30^{\circ} \mathrm{C} / 25^{\circ} \mathrm{C}$ conditions were occupied by SM-like and PM-like tissues; a normal PL was rarely observed (Fig. 4E). Furthermore, its vascular bundle rows disappeared, implying that the organ was transformed into an mrp-like organ. In contrast, in $s p w 1$-cls 1 lodicules formed under $20^{\circ} \mathrm{C} / 20^{\circ} \mathrm{C}$ conditions, the SM-like tissue in spw1-cls 1 lodicules formed under $30^{\circ} \mathrm{C} / 25^{\circ} \mathrm{C}$ conditions was replaced by fibrousparenchymatous intermediate tissue with increased cell size and decreased cell density (Fig. 4F orange arrow). Moreover, blue-stained PL were observed. This organ was a lodicule-mrp intermediate organ, not a complete lodicule, because vascular bundle rows were not formed.

Therefore, the internal structure of spw1-cls 1 lodicules differed according to temperature. Lodicules formed under high-temperature conditions comprised mrp-like tissues, and those formed under low-temperature conditions showed lodicule-like and mrp-like tissue.

\section{Experiment 2: Critical period for low-temperature-} induced cleistogamy instability

To estimate the period during panicle development that is most sensitive to low temperatures for spwl-cls 1 cleistogamy, and the duration of that period, we conducted experiments using various temperature treatment schedules and artificial climate rooms.

In exp. 2-1, the mean FOR of LT-I, LT-II, LT-III, and LTIV, which involved 1 week of low-temperature treatment, was $0.3 \%, 3.2 \%, 6.2 \%$, and $0.1 \%$, respectively (Table 3 ). Although LT-III showed slightly higher values, the values were low overall. The mean FOR of the continuous 14-day treatment series (LT-I+II, LT-II+III, and LT-III +IV), was $3.3 \%, 67.0 \%$, and $11.7 \%$, respectively. LT-II+III had a higher FOR value than the other two schedules despite having the same duration of low-temperature treatment. LT-III+IV showed a higher FOR value than LT-I+II. In the divided 14day $(7+7$ days) schedules (LT-I+III, LT-II+IV, and LTI+IV), the mean FOR was $1.9 \%, 21.5 \%$, and $1.0 \%$, respectively. LT-II+IV showed the highest FOR in this series but was lower than that of LT-II+III. The positive control plan (LT-all) showed the highest FOR value (83.3\%) in exp. 2-1, and there was no flower opening in the negative control (No-LT).

The mean FOR value of LT-II+III was not significantly different from that of LT-all. In contrast, all 1-week treatment schedules, the LT-I+II and LT-III+IV continuous 14day treatment schedules, and the LT-I+III and LT-I+IV divided 14-day treatment schedules showed a low mean FOR, which was not significantly different from that of NoLT. LT-II+IV showed a medium mean FOR value, which was significantly different from those of LT-all and No-LT, but not to those of LT-III and LT-III+IV.

We observed the same tendency in exp. 2-2 (FOR values are shown in Table 3); that is, the mean FOR of the 1-week treatment schedule was low. Of the continuous 14-day treatment schedules, only LT-II+III showed a high FOR value comparable to that of LT-all. LT-II+III and LT-all were in the high FOR value class, LT-III+IV and LT-II+IV in the middle class, and the other schedules and the controls were in the low FOR value class.

In two tests, 14-day continuous low-temperature treatment involving II and III resulted in the greatest increase in the FOR, and LT-II+IV and LT-III+IV slightly promoted flowering. By contrast, 7-day treatment had little effect, even in II or III. Therefore, II and III are the periods of sensitivity to low temperatures, and relatively long ( $>8$ days) lowtemperature periods are needed to open spwl-cls 1 flowers. Experiment 3: Effect of daily temperature change on the cleistogamous phenotype of spw1-cls1

On sunny days, air temperature rises from dawn, peaks in early afternoon, and then declines. In Experiment 3, we mimicked daily temperature changes, and estimated their effects on the flower opening of spw1-cls1.

In exp. 3-1, the mean FOR and DMT values of 25-all were $5.5 \%$ and $25.0^{\circ} \mathrm{C}$, and those of $25-18$ were $55.3 \%$ and $21.5^{\circ} \mathrm{C}$, respectively (Table 4). About half of $s p w 1-c l s 1$ flowers opened in the 25-18 plot, and the DMT of 25-18 was $3.5^{\circ} \mathrm{C}$ lower than that of the 25 -all plot because of the $18^{\circ} \mathrm{C}$ treatment during the dark period $(12 \mathrm{~h})$. These results confirm that this temperature treatment has enough effect to promote flower opening of $s p w 1-c l s 1$, and this temperature schedule was used in exp. 3-2. Next, we inserted 0, 1, 2, and $4 \mathrm{~h}$ high-temperature periods into the light period of the standard temperature cycle.

In exp. 3-2, the mean FOR of D30-0h, D30-1h, D30-2h, and $\mathrm{D} 30-4 \mathrm{~h}$ was $49.9 \%, 24.0 \%, 13.4 \%$, and $0.3 \%$, respectively (Table 4); these values were significantly different. As the $30^{\circ} \mathrm{C}$ duration increased, the FOR value decreased, and spw1-cls1 showed an almost complete cleistogamous phenotype in the D30-4h plot. The DMTs of the plots were $21.5^{\circ} \mathrm{C}, 21.7^{\circ} \mathrm{C}, 21.9^{\circ} \mathrm{C}$, and $22.3^{\circ} \mathrm{C}$, respectively. Although the DMT of D30-4h was $2.7^{\circ} \mathrm{C}$ lower than that of 25 -all, the FOR of D30-4h was equal to or slightly lower than that of 25-all.

\section{Discussion}

In this study, the flowering frequency of $s p w 1-c l s 1$ differed between northern and southern cultivation sites (Table 1). During the cool summer of 2009, while many spw1-cls1 flowers in Sapporo opened, like those of chasmogamous varieties, the ratio of open flowers gradually decreased at more southerly cultivation sites; complete cleistogamy was observed at Fukuyama and Itoman. This decrease in flowering frequency was correlated with the increase in the 30MT value. In 2010, the summer of which was hotter than that in 2009 , the ratio of open flowers was lower in Sapporo, and there was no flower opening at Joetsu and Tsukuba. The area where $s p w 1-c l s 1$ showed complete cleistogamy was shifted northwards, corresponding to the increase in temperature due to annual climatic fluctuations (Table 1). Therefore, cleistogamy of spwl-cls 1 is thermosensitive. As the temperature decreases, the morphological defect of lodicules decreases in severity (Fig. 3), and the cleistogamous 
phenotype is lost.

We also assessed the mechanism of flower opening in spw1-cls 1 at low temperatures. In Experiment 1, we reproduced a series of lodicule phenotypes similar to those under field conditions in various regions. Among them, the lodicules formed under the $30^{\circ} \mathrm{C} / 25^{\circ} \mathrm{C}$ conditions were phenotypically similar to those formed at Itoman in 2009 (Figs. 3F, 4C, left). The internal region of the lodicule was occupied by SM-like fibrous tissue and PM-like parenchyma (Fig. 4E). This lodicule lost its identity like the palealike organ of spw1-1 (Nagasawa et al. 2003) and would fail to push out the glumes by swelling. In contrast, lodicules in the $20^{\circ} \mathrm{C} / 20^{\circ} \mathrm{C}$ plot showed a mild phenotype, which resembled that of Sapporo in 2009 (Figs. 3B, 4C right). Compared to the spwl-cls 1 lodicule in the $30^{\circ} \mathrm{C} / 25^{\circ} \mathrm{C}$ plot, that of $20^{\circ} \mathrm{C} / 20^{\circ} \mathrm{C}$ showed a different internal structure; that is, normal-PL-like parenchymal cells were formed and the number of SM-like fibrous cells was decreased (Fig. 4F). These lodicules are considered to partially restore the lodicule identity and likely possessed swelling function similarly to the ones formed in Sapporo. We can relate a previous molecular analysis of SPW $1^{145 \mathrm{~T}}$ (Yoshida et al. 2007) to the in planta phenotypic change of spwl-cls1. Under hightemperature conditions (e.g., around $30^{\circ} \mathrm{C}$ or higher), because of the decreased ability of SPW $1^{145 \mathrm{~T}}$ to interact with MADS2 or MADS4, the spw1-cls1 lodicule fails to form normal tissue, and instead forms mrp-like tissue, causing the cleistogamous phenotype. In contrast, the ability of SPW $1^{145 \mathrm{~T}}$ to participate in protein-protein interactions was partially restored under low-temperature conditions (e.g., around $20^{\circ} \mathrm{C}$ or lower), resulting in the formation of normal-lodicule-like tissue in the spwl-cls 1 lodicule. The amount of normallodicule-like tissue would increase with decreasing temperature, and the proportion of mrp-like and normal-lodiculelike tissue in the lodicule would determine whether the flower opens or not.

\section{The temperature sensitivity of spw1-cls1 implies the lodi-} cule forming stage

Because the suppression of spw1-cls1 cleistogamy is caused by alteration of the internal structure of lodicules, we hypothesized that there is a thermosensitive period before heading, especially during lodicule formation. In Experiment 2, the FOR was increased by the 14-day continuous low-temperature treatment through the periods from II to III. In addition, the FOR was higher in the LT-II+IV and LT-III+IV schedules. Combined with the results of Experiment 1 and our previous yeast two-hybrid analysis (Yoshida et al. 2007), the marked effect of the low-temperature treatments in II and III on the FOR of spw1-cls 1 may be because lodicule identity and cellular organization are established by the restored activity of the SPW1-MADS2/4 protein complexes during these periods. Although the treatment in IV influenced flower opening, the FOR was lower than that in II and III. This implies that lodicule formation is almost complete in this period. In contrast, the treatments that in- cluded I (e.g., LT-I+II, LT-I+III, etc.) had little effect on the FOR (Table 3). Ikeda et al. (2004) categorized and organized the developmental stages of rice inflorescence and spikelet. While the beginning of I corresponds to stage In5, lodicule formation does not start at this stage but at stage In7 (more specifically, stage Sp5). SPW1 expression is initiated in Sp5 (Ikeda et al. 2004); therefore, the negligible effect of low-temperature treatment in I on the FOR is reasonable. This result indirectly supports a relationship between low-temperature treatment and the behavior of SPW $1^{145 T}$. The low-temperature treatments in single II, III, and IV had little effect on flower opening. Therefore, the lowtemperature duration was insufficient to generate enough cells for normal-PL-like tissue, which enables flower opening. The low-temperature treatments in both II and III resulted in recovery of lodicule identity and maintenance of cellular development, and were sufficient to cause flower opening. Although the critical duration of low-temperature treatment may vary depending on the temperature, the results of Experiment 2 indicated that relatively short ( $\leq 7$ days) low-temperature periods do not result in opening of flowers in spwl-cls 1 .

In Experiment 3, a relatively short high-temperature period in the daytime reduced the low-temperature-mediated promotion of flower opening of spw1-cls1. These results imply that, under natural conditions, the cleistogamy of spw1-cls 1 is maintained in areas with a sufficiently high daytime temperature ( $\sim \mathrm{h}$ in the early afternoon). Further experiments are needed to clarify the balance and duration of daytime and nighttime temperatures required to maintain cleistogamy.

$S P W 1$ is expressed in the lodicule primordium and is maintained in the mature lodicule (Nagasawa et al. 2003, Ohmori et al. 2009). Therefore, we speculate that SPW1 expression in the lodicule primordium of WT spikelets specifies lodicule identity and maintains cellular integrity, as normal lodicules require continuous expression and sufficient activity of the SPW1-MADS2/4 complex. However, the protein-protein interaction between $\mathrm{SPW} 1^{145 \mathrm{~T}}$ and MADS2 or MADS4 is temperature-sensitive and is weaker at high (e.g., $\left.28^{\circ} \mathrm{C}\right)$ than at low (e.g., $20^{\circ} \mathrm{C}$ ) temperatures (Yoshida et al. 2007). Therefore, $30^{\circ} \mathrm{C}$ would result in dissociation of the SPW $1^{\mathrm{I} 45 \mathrm{~T}}$-MADS2/4 dimers that formed during the low-temperature treatment. This associationdissociation of the SPW $1^{\text {I45T }}$-MADS2/4 dimer likely causes phenotypic plasticity in spw1-cls 1 lodicules, with intermediate identities between normal lodicules and the mrp-like organ. Indeed, we observed a mixture of lodicule-like, mrplike, and intermediate tissues in the spw1-cls1 lodicule in Experiment 1 (Fig. 4F). Because autoregulatory circuits of B-class genes has been already reported in other plants (Goto and Meyerowitz 1994, Jack et al. 1994, Yang et al. 2003, Zachgo et al. 1995), downregulation of SPW1, MADS2 and MADS4 caused by the reduced activity of the SPW $1{ }^{145 T}$-MADS2/4 complex can be expected in rice and may enhance the phenotype of spw1-cls 1 . Examination of 
the transcriptional regulation of these genes would help further understanding the developmental mechanism of rice lodicule and the utilization of $s p w 1-c l s 1$.

\section{Use of spw1-cls1 for suppression of outcrossing}

To combine our findings (i.e. loss of the cleistogamous phenotype of spwl-cls1 due to low temperatures and the thermosensitive period) with climatic data at the cultivation sites, we could simulate the phenotypes of $s p w 1-c l s 1$, and predict the areas where the cleistogamous phenotype of spw1-cls1 would be maintained. For this, the mean temperature data before the expected heading date would be useful. However, our results imply that the mean temperature is not necessarily correlated with the $s p w 1-c l s 1$ phenotype. For example, the FORs of D30-4h and 25-all in Experiment 3 were not markedly different despite their significantly different DMTs. In addition, although the different conditions of the two experiments should be taken into consideration, it is interesting to compare the results of the LT-all schedule in Experiment 2 and the D30-4h plot in Experiment 3. LT-all involved $24^{\circ} \mathrm{C} / 20^{\circ} \mathrm{C}$ for 4 weeks before heading, and the DMT was $22.0^{\circ} \mathrm{C}$. In contrast, the DMT of D30-4h was $22.3^{\circ} \mathrm{C}$, which is similar to that of LT-all. However, the phenotypes in LT-all and D30-4h were different. This result implies that we should take into consideration not only the mean temperature but also the daily highest temperature and its duration. Further investigation is necessary to establish a method of simulating spwl-cls 1 phenotype and predicting suitable areas.

The stability of cleistogamy is important for inhibition of outcrossing by pollen dispersal. Our results imply that care is needed if the cleistogamy of $s p w 1-c l s 1$ is used to inhibit outcrossing in areas with a cool summer. However, the FOFs of spwl-clsl were significantly lower than those of the WT lines in all areas, except in Sapporo. A low FOF value implies release of only a small amount of pollen into the air, which may not cause outcrossing and may be sufficient for gene containment because the original outcrossing rate of cultivated rice is low. Of course, it should be taken into account that low temperatures before heading would cause male sterility and increase the outcrossing rate (Sato and Yokoya 2008, Tanno et al. 2011). Further experiments are needed to clarify these issues.

The cleistogamy of rice is likely to become more important. Outcrossing between glutinous and non-glutinous lines has been an issue of concern because it results in loss of the glutinous trait (Kamagata et al. 1988, 1990). To suppress outcrossing during co-cultivation of these types of rice, a spatial barrier (sufficient distance between cultivated paddy fields) or temporal barrier (different heading date for each cultivar by shifting the cultivation period) can be employed, but more space or effort is required. In addition, a non-GM rice cultivar with herbicide resistance has been outcrossed with weedy rice, resulting in weedy rice with herbicide resistance (Gealy et al. 2015). Pollen-mediated gene flow from cultivated rice to weedy rice is a concern not only in GM rice but also in non-GM rice. Inhibition of outcrossing by cleistogamy is a practical solution for these issues. In contrast, Koike et al. (2015) reported that cleistogamous rice is resistant to male sterility caused by high-temperature stress at flowering time because the inner temperature of closed flowers is maintained at a lower level than the outer temperature, and the pollen maintains its germination activity. It is possible that rice cleistogamy can prevent the yield loss caused by high-temperature stress due to global warming.

A novel rice cleistogamous $s p w 1-c l s 2$ mutant was reported (Lombardo et al. 2017). spw1-cls2 has a missense mutation at a different position in SPW1 compared with spw1-cls1, and its mechanism of cleistogamy differs from that of $s p w 1$ cls1. The advantage of $s p w 1-c l s 2$ is non-thermosensitive cleistogamy; that is, there is no climatic limitation on the cultivation region to inhibit outcrossing. However, the fertility of $s p w 1-c l s 1$ is superior to that of $s p w 1-c l s 2$. Therefore, $s p w 1-c l s 1$ is preferable to $s p w 1-c l s 2$ in suitable areas. In this study, we showed that the cleistogamy of spwl-cls 1 is a thermosensitive character and linked the molecular mechanism of SPW $1^{145 \mathrm{~T}}$ with lodicule phenotype at various temperatures. We also determined the critical period sensitive to low temperatures, and the response to daily changes in temperature. These results will facilitate the use of climatic data to simulate the flowering behavior of spwl-cls 1 and predict suitable cultivation areas. At present, spwl-cls 1 is the only source of cleistogamy confirmed to have the ability to contain gene flow (Ohmori et al. 2012). If the climatic conditions critical for maintaining spw1-cls1 cleistogamy are determined, the mutation can be used to inhibit outcrossing.

\section{Acknowledgments}

We thank Y. Sato, M. Yamaguchi, R. Kaji, K. Nakagomi, K. Nagano, T. Endo, M. Ohshima, K. Ashida and N. Takamine for great contribution to experimental cultivation in various regions in Japan. We also thank K. Tsukada, K. Yukawa, $\mathrm{N}$. Ichimura, and N. Uesaka for their helpful assistance in various experiments, K. Hayashi, M. Kimizu, H. Tabuchi and $\mathrm{O}$. Yatou for helpful discussion, and T. Genba, S. Yuminamochi, K. Koide, K, Yazaki and S. Saito for their help in the rice cultivation. This work was supported by a grant from the Ministry of Agriculture, Forestry and Fisheries of Japan (Research Project for Genomics for Agricultural Innovation).

\section{Literature Cited}

Daniell, H. (2002) Molecular strategies for gene containment in transgenic crops. Nat. Biotechnol. 20: 581-586.

Endo, T., H. Sato, M. Yamaguchi, T. Kataoka, K. Nakagomi, T. Ito and K. Mori (2009) Estimate of outcrossing rates in a rice plant (Oryza sativa L.) under field conditions using a purple grain rice cultivar, Okunomurasaki. Breed. Sci. 59: 195-202.

Gealy, D.R., N.R. Burgos, K.M.Yeater and A.K. Jackson (2015) Outcrossing potential between U.S. blackhull red rice and indica rice 
cultivars. Weed Sci. 63: 647-657.

Goto, K. and E.M. Meyerowitz (1994) Function and regulation of the Arabidopsis floral homeotic gene PISTILLATA. Genes Dev. 8: $1548-1560$.

Gressel, J. (2012) Containing and mitigating transgene flow from crops to weeds, to wild species, and to crops. In: Altman, A. and P.M. Hasegawa (eds.) Plant Biotechnology and Agriculture: Prospects for the 21st Century, Elsevier, Amsterdam, pp. 509-523.

Han,A., P.Fan, J.C.Stroud, H.D. Youn, J.O.Liu and L.Chen (2003) Sequence-specific recruitment of transcriptional co-repressor Cabin1 by myocyte enhancer factor-2. Nature 422: 730-734.

Hoshikawa, K. (1989) The growing rice plant. Nobunkyo, Tokyo, pp. 14-15, 240-245.

Hüsken,A., S. Prescher and J. Schiemann (2010) Evaluating biological containment strategies for pollen-mediated gene flow. Environ. Biosafety Res. 9: 67-73.

Ikeda, K., H. Sunohara and Y. Nagato (2004) Developmental course of inflorescence and spikelet in rice. Breed. Sci. 54: 147-156.

ISAAA (2016) Global Status of Commercialized Biotech/GM Crops: 2016. ISAAA, New York, Brief No. 52.

Jack, T., G.L.Fox and E.M. Meyerowitz (1994) Arabidopsis homeotic gene APETALA3 ectopic expression: transcriptional and posttranscriptional regulation determine floral organ identity. Cell 76: 703-716.

Kamagata, T., R.Hasegawa, T.Hatakeyama and J.Fujishiro (1988) Studies on the reversion of glutinous seeds to non-glutinous seeds in seed production culture of paddy rice. I. Mechanism of the reversion and present measures. Bull. Chiba Found. Seed \& St. Farm 10: $13-25$.

Kamagata, T., T. Hatakeyama and J.Fujishiro (1990) Studies on the reversion of glutinous seeds to non-glutinous seeds in seed production culture of paddy rice. II Varieties and preventive measures against natural outcrossing. Bull. Chiba Found. Seed \& St. Farm 12: $1-7$.

Kato, H. and H. Namai (1987a) Intervarietal variations of floral characteristics with special reference to $F_{1}$ seed production in japonica rice (Oryza sativa L.). Japan. J. Breed. 37: 75-87.

Kato, H. and H. Namai (1987b) Floral characteristics and environmental factors for increasing natural outcrossing rate for $F_{1}$ hybrid seed production of rice (Oryza sativa L.). Japan. J. Breed. 37: 318-330.

Kawakatsu, T., J. Itoh, K. Miyoshi, N. Kurata, N.Alvarez, B. Veit and Y.Nagato (2006) PLASTOCHRON2 regulates leaf initiation and maturation in rice. Plant Cell 18: 612-625.

Koike, S., T. Yamaguchi, S. Ohmori, T. Hayashi, O. Yatou and H. Yoshida (2015) Cleistogamy decreases the effect of high temperature stress at flowering in rice. Plant Prod. Sci. 18: 111-117.

Lombardo, F., M. Kuroki, S.G. Yao, H. Shimizu, T. Ikegaya, M. Kimizu, S. Ohmori, T.Akiyama, T. Hayashi, T. Yamaguchi et al. (2017) The superwoman1-cleistogamy2 mutant is a novel resource for gene containment in rice. Plant Biotechnol. J. 15: 97-106.

Maeng, J.Y., Y.J.Won, R.Piao, Y.I.Cho, W.Jiang, J.H.Chin and H.J. Koh (2006) Molecular mapping of a gene ' $l d(\mathrm{t})$ ' controlling cleistogamy in rice. Theor. Appl. Genet. 112: 1429-1433.

Mallory-Smith, C. and M. Zapiola (2008) Gene flow from glyphosateresistant crops. Pest Manag. Sci. 64: 428-440.

Marathi, B. and K.K. Jena (2015) Floral traits to enhance outcrossing for higher hybrid seed production in rice: present status and future prospects. Euphytica 201: 1-14.

Messeguer, J., C.Fogher, E. Guiderdoni, V.Marfà, M.M.Català, G.
Baldi and E. Melé (2001) Field assessments of gene flow from transgenic to cultivated rice (Oryza sativa L.) using a herbicide resistance gene as tracer marker. Theor. Appl. Genet. 103: 11511159 .

Nagao, S. and M. Takahashi (1954) Genetical studies on rice plant. XVIII. Some histological and genetical observations on cleistogamous spikelets. Japan. J. Breed. 4: 135-139.

Nagasawa, N., M. Miyoshi, Y. Sano, H. Satoh, H. Hirano, H. Sakai and Y. Nagato (2003) SUPERWOMAN1 and DROOPING LEAF genes control floral organ identity in rice. Development 130: 705-718.

Ni, D.H., J.Li, Y.B.Duan, Y.C.Yang, P.C.Wei, R.F.Xu, C.R.Li, D.D. Liang, H. Li, F.S. Song et al. (2014) Identification and utilization of cleistogamy gene $c l 7(t)$ in rice (Oryza sativa L.) J. Exp. Bot. 65: 2107-2117.

Norman, C., M. Runswick, R. Pollock and R. Treisman (1988) Isolation and properties of cDNA clones encoding SRF, a transcription factor that binds to the c-fos serum response element. Cell 55: 9891003.

Ohmori, S., M. Kimizu, M. Sugita, A. Miyao, H. Hirochika, E. Uchida, Y. Nagato and H. Yoshida (2009) MOSAIC FLORAL ORGANS1, an $A G L 6$-Like MADS box gene, regulates floral organ identity and meristem fate in rice. Plant Cell 21: 3008-3025.

Ohmori, S., H. Tabuchi, O. Yatou and H.Yoshida (2012) Agronomic traits and gene containment capability of cleistogamous rice lines with the superwoman1-cleistogamy mutation. Breed. Sci. 62: 124132.

Phan, P.D.T., H. Kageyama, R. Ishikawa and T. Ishii (2012) Estimation of the outcrossing rate for annual Asian wild rice under field conditions. Breed. Sci. 62: 256-262.

Rong, J., B.R. Lu, Z. Song, J. Su, A.A. Snow, X.Zhang, S. Sun, R. Chen and F. Wang (2007) Dramatic reduction of crop-to-crop gene flow within a short distance from transgenic rice fields. New Phytol. 173: 346-353.

Rong, J., F. Wang, Z.Song, J.Su, R. Chen and B.R.Lu (2012) Scale effect on rice pollen-mediated gene flow: implications in assessing transgene flow from genetically engineered plants. Ann. Appl. Biol. 161: 3-11.

Satake, T. (1972) Circular dense-culture of rice plants in pots, the purpose of obtaining many uniform panicles of main stems. Jpn J. Crop Sci. 41: 361-362.

Sato, Y. and S. Yokoya (2008) Effects of male sterility caused by low temperature at the booting stage on out-crossing rates in rice (Oryza sativa L.). Breed. Res. 10: 127-134.

Tanno, H., T. Takeuchi, H. Kiuchi and H. Shibaike (2011) Relation between out-crossing rate and isolation distance under cool temperature conditions at the booting stage that causes the occurrence of male sterility in rice. Jpn J. Crop Sci. 80: 49-58.

Yang, Y., H.Xiang and T.Jack (2003) pistillata-5, an Arabidopsis B class mutant with strong defects in petal but not in stamen development. Plant J. 33: 177-188.

Yoshida, H., J.Itoh, S. Ohmori, K. Miyoshi, A. Horigome, E. Uchida, M.Kimizu, Y.Matsumura, M.Kusaba, H. Satoh et al. (2007) superwoman1-cleistogamy, a hopeful allele for gene containment in GM rice. Plant Biotechnol. J. 5: 835-846.

Zachgo, S., E.D.A. Silva, P.Motte, W.Tröbner, H. Saedler and Z. Schwarz-Sommer (1995) Functional analysis of the Antirrhinum floral homeotic DEFICIENS gene in vivo and in vitro by using a temperature-sensitive mutant. Development 121: 2861-2875. 